Center for

Mathematical Economics

Working Papers

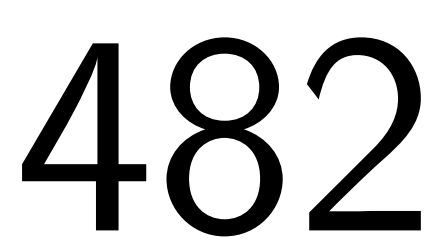

May 2013

\title{
The LIBOR mechanism and Related Games
}

Christoph Diehl

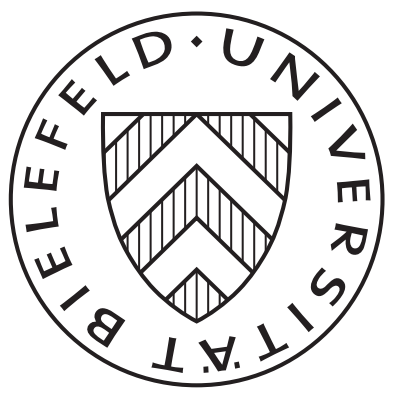

Center for Mathematical Economics (IMW) 


\title{
The LIBOR mechanism and Related
}

\section{Games}

\author{
Christoph Diehl 1
}

Bielefeld University and Université Paris 1 Panthéon-Sorbonne

This Version: May 31, 2013

\begin{abstract}
The London InterBank Offered Rate (LIBOR) is the most important set of interest rate benchmarks. Recently there have been reports about systematic manipulation of the LIBOR. We thus investigate incentives and possibilities to rig the LIBOR or related statistics for quote submitting panel banks. Both reputation concerns and financial exposure to the index may lead to misrepresentation of borrowing costs. Even in the static model we consider, we show that incorrect quoting is the standard and honesty the exception. In particular, we can theoretically explain why the LIBOR quotes were too low during the financial crisis which started in 2007, when increasing panel bank sizes is helpful and why individual quotes should be published with delay. Moreover, we evaluate and compare the performance of different aggregators like the median, the trimmed average and the average.
\end{abstract}

\footnotetext{
${ }^{1}$ Center of Mathematical Economics, Bielefeld University, christoph.diehl@uni-bielefeld.de.

I thank Frank Riedel and Jean-Marc Tallon for most useful comments, discussions and ideas.

Financial support by the International Research Training Group EBIM, "Economic Behavior and Interaction Models", the Franco-German University (DFH-UFA) and by the Center of Mathematical Economics at Bielefeld University is gratefully acknowledged.
} 


\section{Introduction and Motivation}

"This dwarfs by orders of magnitude any financial scams in the history of markets" Comment on the LIBOR scandal by Andrew Lo, MIT Professor of Finance, 2012.

The London InterBank Offered Rate (LIBOR) is the most important set of interest rate benchmarks in the financial world. It is estimated that it affects more than $\$ 350$ trillion worth of derivatives, flexible mortgage rates and student loans. Almost everybody is directly affected by changes of the LIBOR. The BBC has called it "the most important number in the world". 22 The British Bankers' Association writes "The London Interbank Offered Rate (LIBOR) is the primary benchmark for short term interest rates globally. 3 In the last months reports about systematic manipulation of the LIBOR have emerged (this is written May 2013). These reports necessitate a systematic investigation of the current LIBOR mechanism in a theoretical model to identify the strengths and weaknesses of the mechanism and the possibilities of banks to manipulate the index.

For this end, we consider several strategic games which are closely related to the actual LIBOR administering process. The LIBOR is currently calculated as the trimmed average of submitted quotes of banks which belong to the LIBOR panel. The top and bottom $25 \%$ of the quotes are deleted, the remaining quotes are averaged, the result is the LIBOR. Published are however all quotes of all banks. The quotes of the banks do not have to be based on real transactions, instead banks answer the question: "At what rate could you borrow funds, were you to do so by asking for and then accepting interbank offers in a reasonable market size just prior to 11am?" This opens the door to manipulation of the index. We will coin the name "the LIBOR problem" for the problem of building a mechanism and using a statistic such that the resulting index based on the submitted quotes is the same as if the index were calculated using the private values of the banks. Different statistics besides the trimmed average are the median and the average of all quotes. We will formulate games which are identical except for the statistic that is used to aggregate the individual quotes. The statistic enters the utility function of the strategically acting banks, the players in the game.

\footnotetext{
2 http://news.bbc.co.uk/2/hi/business/7680552.stm

3 http://www.bbalibor.com/bbalibor-explained/faqs
} 
Our main findings are the following. Acting strategically, the default quote of a bank is not the true quote, except when the bank cannot influence the statistic. In the Average Game, banks thus always misrepresent except reputational concerns and financial incentives exactly balance. In this game, an increase in the panel bank size decreases the magnitude of misrepresentation if reputational concerns are not important for the banks. We find somewhat different results in the Median Game. While it remains true that banks either quote honestly or such that they influence the median, for uneven $n$ there always exists a player who quotes truthfully. This does not hold for even $n$. For the case $n=3$ three different types of equilibria arise, depending on the distribution of the private values and the financial exposure of the banks. A truthtelling equilibrium may arise, an indeterminate equilibrium set where a majority of banks controls the median by quoting the same, and an equilibrium in which only one bank misrepresents while the others quote truthfully. The notions of trimmed average, median and average are only distinct if $n \geq 5$. We present a theorem which delivers a comparison between the maximal misrepresentation in equilibrium in the three games. Due to the different marginal impact of the players which influence the statistic, the misrepresentation can be largest in the Median Game, followed by the Trimmed Average Game and is lowest in the Average Game. Additionally, we prove the existence of a maximal misrepresentation equilibrium for all considered games. This theorem holds independently of the number of players.

For the formulation of the different, LIBOR-related games we suggest a particular kind of utility function which captures the incentive structure of the banks. Different factors influence the final quotation. We argue that the utility function has to capture the financial exposure of the bank to the LIBOR or the respective statistic. Moreover, there may be reputational concerns the banks face, in particular during a financial crisis. As all quotes of all banks are published, high quotes relative to the other quotes or relative to the index may indicate a certain weakness of the financial institution which may entail certain risks like an increased probability of a bank run. Finally, we argue for an anchoring of the bank's quote at the private value. Misquoting is not legal and if detected is punished. Barclays had to pay more than $\$ 400$ million for the settlement of charges and UBS more than $\$ 1$ billion for having tried to manipulate the LIBOR. As the reputational concerns only point in the direction of lower quoting and arguably only play an important role in times of financial turmoil and the actual manipulation goes way back before the financial crisis of 2007, we focus in large parts of the paper on financial misrepresentation 
incentives and the anchoring of the costs in the utility function.

We opt for a linear quadratic structure of the utility function, close to the utility function choices of Chen (2012) and Snider and Youle (2010), the most important other contributions in the very small theoretical literature on the LIBOR mechanism. We defend this choice in detail in section 3. Another important assumption that we make is that all players know the private values of all players, while the mechanism designer, the institution that administers the LIBOR, has no information about these private values and only observes what the players actually quote. We do not claim that this strong information asymmetry accurately describes the real world. However, from our perspective it is reasonable to assume that banks are better informed about the actual borrowing costs of other banks than the regulating authority. This may be due to the fact that panel banks are similarly structured and are actually operating on the interbank market and are not only observing it. Nevertheless, this remains a simplification.

The literature which investigates the LIBOR, the quotation patterns, and in particular the possibilities of and indications for manipulation, is rapidly growing. At the moment there are still relatively few contributions. There exist several notable empirical investigations but only two papers which formulate a theoretical model and directly address the LIBOR problem. We start by summarizing the empirical literature.

The first paper which investigated the LIBOR quotes for suspicious patterns was Abrantes-Metz et al. (2012). They used a screen for collusion and found dubious quote distributions which may indicate manipulation attempts. Abrantes-Metz et al. (2011) use a different approach and apply a test based on Benford's law to also find evidence for possible fraud, as the quote distribution they find is highly suspicious. The approach of Kuo et al. (2012) is to examine different indicators for the borrowing costs of banks like the inferred term borrowing costs derived from FedWire, a data system for interbank borrowing costs based on real transactions. They come to the conclusion that the LIBOR quotes are 10-30 basis points lower than the comparison rates in the times of huge financial turmoil after the collapses of Bear Sterns and Lehman Brothers. In other times, the different rates are statistically indistinguishable. In a new paper by Eisl et al. (2013) EURIBOR and LIBOR fixings are investigated and their performance is compared to alternative rate fixings like the median, the mean, and a randomized procedure. They find that the manipulability for the median is much lower provided the panel of banks is sufficiently large 
and thus recommend switching from the trimmed average to the median.

The theoretical literature on the LIBOR mechanism is still small, but due to the ongoing LIBOR scandal a rapid growth of the literature is expected. The first who investigated the LIBOR in a theoretical model were Snider and Youle (2010). They sketch a model which predicts quote clustering of the quotes of the banks around the pivotal $25 \%$ and $75 \%$ quantile. As the LIBOR is calculated on a daily basis and quotes normally do not differ a lot from the previous day, banks have a good knowledge of the next quote of their competitors. Thus, they are able to accurately predict the interval of quotes which are taken for the actual LIBOR calculation and can optimize the position of their own quote inside this interval which entails the quote clustering at the borders. Snider and Youle (2010) then look at data and find evidence supporting their theoretical predictions. In particular, they find indications for fraud not only during the times of market turmoil, but also before and after. Interestingly, the first version of their paper has been written in 2009 and thus predates the revelations in the LIBOR scandal.

A second important contribution is the paper by Chen (2012) which is also still unpublished. She works in a Bayesian framework where the realization of the private value is private knowledge of the respective bank. The distribution of these private values is iid and known to all players and the mechanism designer. She suggests a similar utility function to ours but only considers the incentive for banks to lower their own quote. In this sense, she is primarily concerned with reputational incentives of the banks. She investigates the best responses of the players and suggests a mechanism to solve the LIBOR problem which is in the spirit of d'Aspremont and Gérard-Varet (1979). She then looks at data and calibrates the parameters in her utility function.

Our paper is organized as follows. We start by giving background on the LIBOR. We describe the origins of the index, how it became the most important benchmark index in the financial industry and how it is calculated and administered. We then turn to the LIBOR scandal, the attempted systematic manipulation of the index by the panel members. The scandal really unfurled in June / July 2012 and the magnitude of the scandal is still hard to estimate. However, reports on the manipulation go back to the earliest days of the LIBOR. We go on to describe policy answers to the LIBOR problem, in particular the suggestions of the Wheatley review by Wheatley (2012a), and speculate on the future of the LIBOR as a benchmark index. We 
present our baseline model of a strategic game in section 3 before we investigate the different LIBOR-related games in section 4. The games only differ in the way the quotes are aggregated. In the conclusion we point out how our findings may add to the understanding of the LIBOR mechanism and the evaluation of suggested policy approaches on the fixing of the LIBOR problem.

\section{Background on the LIBOR}

In this section we give background on the London Interbank Offered Rate. Starting with a historical perspective, we continue with a description of the current LIBOR mechanism before we present a detailed chronology of the LIBOR scandal and policy approaches in response to the LIBOR scandal. Besides the cited academic articles we draw primarily from newspaper articles and give the references in the footnotes.

\subsection{Inception and historical development of the LIBOR}

The London InterBank Offered Rate was institutionalized in 1986. The origins of the rate however go back to the late 1960s. In the early days of the LIBOR, the fixing of the rate can best be described as gentlemen's agreement between likeminded bankers. I In that form it was set and governed by a small group of bankers in London between the late 1960s and the early 1980s. The very first LIBOR loan was a $\$ 80$ million loan by a group of banks to Iran. The banker at Manufacturers Hanover, Minos Zombanakis, who was responsible for the loan, had to come up with a rate fixing. Thus he called several banks in London and asked them to send him their cost of money. He used a trimmed average of the set of rates that were handed in and called it the London InterBank Offered Rate. For the next 15 years the rate was set in a similar way: Banks were asked for their costs of money and a trimmed set of the numbers handed in were arithmetically averaged to fix the LIBOR.

Starting from 1984, the way the LIBOR was governed changed. New financial instruments like

\footnotetext{
${ }^{4}$ Landon Thomas Jr., Trade Group for Bankers Regulates a Key Rate, July 05, 2012, New York Times
} 
interest rate swaps emerged and banks started to trade heavily in these new areas. Banks were worried that nonuniformity and the adhoc structure of the LIBOR calculation would hinder the growth of the new markets for banks. A demand for an institutionalization of the LIBOR arose. Driven by this demand, the British Bankers' Association (BBA) presented the BBA standard for interest rate swaps (BBAIRS), the predecessor of the LIBOR, in 1985. The BBA LIBOR officially commenced on 1.1.1986 and immediately became standard market practice. There have been some minor modifications in the way the LIBOR is governed and calculated since 1986. The composition of the panels has changed frequently and new tenors and currencies have been added. Importantly, the LIBOR question has been changed in 1988. Up to 1988, the question was: "At what rate do you think interbank term deposits will be offered by one prime bank to another prime bank for a reasonable market size today at 11am?" The question was changed to: "At what rate could you borrow funds, were you to do so by asking for and then accepting interbank offers in a reasonable market size just prior to 11am?" The new formulation was chosen to enable accountability for the rates, to cite the BBA. Interestingly, the LIBORquestion differs from the EURIBOR-question. The EURo InterBank Offered Rate is also a survey based set of interest rates. The EURIBOR-question is: "Contributing panel banks must quote the required euro rates to the best of their knowledge; these rates are defined as the rates at which euro interbank term deposits are being offered within the EMU zone by one prime bank to another at 11.am Brussels time." An analysis of the importance of the two different questions can be found in Eisl et al. (2013). Important to note is that the main idea of asking banks for their borrowing costs and using their answers to calculate a number, the LIBOR, has not been changed since the 1960s.

The significance of the LIBOR has massively increased in the last years and decades. To get an idea of the centrality that the LIBOR plays in the financial world it is best to look at some numbers. It is estimated that contracts with notional value of more than $\$ 350$ trillion are linked to the LIBOR. More than $50 \%$ of all mortgage contracts in the United States have flexible mortgage rates which depend on the LIBOR. Recent documents show that Deutsche Bank has made more than 500 million Euro with bets on the LIBOR only in 2008. 5 The same documents give the information that as of September 30, 2008, Deutsche Bank could "gain or lose as much as about 68 million for each one-hundredth of a percentage point change in the

\footnotetext{
$\sqrt[5]{\text { http://online.wsj.com/article/SB10001424127887324442304578231721272636626.html }}$
} 
gap between different rates related to Libor and the euro interbank offered rate". With other words, the importance of LIBOR is massive and can hardly be underestimated. A possible manipulation of the LIBOR is of enormous financial interest for the banks. Before we give a chronology of the LIBOR scandal, in which the banks apparently indeed rigged or tried to rig the LIBOR, we first summarize the administering process.

\subsection{Administering of the LIBOR}

In this section we describe how the LIBOR is currently administered (May 2013). At the moment the LIBOR is published daily in 15 different maturities (overnight, 1 week, 2 weeks, 1 month, 2 months, ..., 12 months) and 10 different currencies (Pound Sterling, US Dollar, Japanese Yen, Swiss Franc, Canadian Dollar, Australian Dollar, Euro, Danish Kroner, Swedish Krona, New Zealand Dollar). For all currencies and maturities there exist panels which consist of 6-18 members. These members are chosen by the British Bankers' Association (BBA), the organization responsible for the administering process, according to the three criteria scale of market activity, credit rating, and perceived experience in the currency concerned. Panel bank members include the large European, American and Asian banks like Deutsche Bank, UBS, Barclays, Bank of America, Mitsubishi etc. Some of the panel banks like Barclays and HSBC are themselves member of the BBA, thus had to oversee themselves. Interestingly, Marcus Agius, who was chairman of Barclays till the revelations of the LIBOR scandal of June 2012, was also chairman of the BBA. He stepped down from this position when he stepped down from his chairman position at Barclays.

The most important single LIBOR rate is the three months US Dollar which is fixed by a panel of currently 18 banks. The actual calculation of the LIBOR is done by the data provider Thomson and Reuters. All panel banks are given a special application which allows the banks to secretly transfer the daily rates for the respective currencies and tenors to Thomson and Reuters. The time window for this transfer is 11-11.10am GMT. In this time window, banks submit their set of quotes which is their answer to the question "At what rate could you borrow funds, were you to do so by asking for and then accepting interbank offers in a reasonable market size just prior to 11am?" After having received the data packages from the banks, Thomson 
and Reuters performs a series of statistical tests on the received data sets to ensure no mistakes have been made by the submitting banks. The actual LIBOR rates are then calculated and together with all original quotes of all banks published by Thomson and Reuters and other licenced data vendors at precisely $12 \mathrm{am}$ GMT.

\subsection{The LIBOR scandal}

The LIBOR scandal started to really unfurl in June / July 2012 when the first major bank, Barclays, admitted that it had submitted quotes for the LIBOR calculation which did not accurately reflect their own perception of their interest rate they would have to pay to borrow on the unsecured interbank market. They agreed to pay a penalty of $\$ 450$ million for a settlement. However, reports on systematic manipulation of the LIBOR go back far longer. The Wall Street Journal published a series of articles in April and May 2008 which questioned the accurate quoting of banks. On April 16, 2008, ${ }^{6}$ the journal printed the article "Bankers cast doubt on key rate amid crisis" by Carrick Mollenkamp where it was stated that "One of the most important barometers of the world's financial health could be sending false signals". The article cited several bankers and the report by Gyntelberg and Wooldridge (2008) which expressed the concern that banks may quote incorrectly. The sources quantified the misrepresentation to be 20-30 basis points below the actual borrowing costs. The article already mentioned the two main reasons for misrepresentation of true borrowing costs. First, "Some banks don't want to report the high rates they're paying for short-term loans because they don't want to tip off the market that they're desperate for cash". In other words, banks submit lower quotes to appear financially stronger. Reputational concerns drive the misrepresentation. The report by Gyntelberg and Wooldridge (2008) suggested that "banks might have an incentive to provide false rates to profit from derivatives transactions." Thus, the second reason for misrepresentation are financial incentives. In a follow up article published in the Wall Street Journal on May 29, 2008, the reporters Mollenkamp and Whitehouse analyzed in detail the quoting patterns of banks and came to the conclusion that some banks may have submitted quotes during the financial crisis which were too low and thus indicated a false financial situation of the banks. 7

\footnotetext{
${ }^{6}$ http://online.wsj.com/article/SB120831164167818299.html

7http://online.wsj.com/article/SB121200703762027135.html
} 
In particular, the study compared the LIBOR submissions to credit default swaps, an indicator of financial strength. This article received a lot of attention and triggered research on the question if the LIBOR is reliable and a credible indicator of the actual borrowing costs of banks. Other financial publications like the Financial Times followed in formulating concerns about the reliability and accurateness of the LIBOR. 8

The British Bankers' Association responded to the reports. Angela Knight, who then was CEO of the BBA, said there is no need to replace or reform LIBOR. She said "I see no reason suddenly to up sticks and change a process that has actually served the financial community worldwide extremely well for a very considerable number of years". Some investigations supported her statement. The already cited Gyntelberg and Wooldridge (2008), a report of the Bank for International Settlements, highlighted incentives for misrepresentation due to financial exposure to the LIBOR. However, "the available data do not support the hypothesis that contributors manipulated their quotes from positions based on fixings." Moreover, in its global financial stability review of October 2008, the IMF came to the conclusion that "Although the integrity of the U.S. dollar Libor-fixing process has been questioned by some market participants and the financial press, it appears that U.S. dollar Libor remains an accurate measure of a typical creditworthy bank's marginal cost of unsecured U.S. dollar term funding." 9 Nevertheless, the reports about inaccurateness and problems with the LIBOR continued. Willem Buiter, then professor at the London School of Economics, wrote in August 2008 in Buiter (2008) that "The unsecured interbank market became illiquid to the point that Libor now is the rate at which banks won't engage in unsecured lending to each other". In November 2008, the governor of the Bank of England, Mervyn King, described the LIBOR before parliament in the following way: "It is in many ways the rate at which banks do not lend to each other, and it is not clear that it either should or does have significant operational content." ${ }^{10}$ Documents released in July 2012 prove that the New York Fed and the Bank of England discussed reforms to the LIBOR setting system as far back as June 2008. ${ }^{11}$ Mervyn King and Timothy Geithner, then head of the New York Fed and later treasury secretary under President Obama, exchanged a series of

\footnotetext{
${ }^{8}$ Mackenzie, M. and Tett, G. 2008. Libor remarks fail to put unease to rest. Financial Times (June 2)

${ }^{9}$ Global Financial Stability Report, World economic and financial surveys (International Monetary Fund):

76. October 2008

${ }^{10}$ Examination of Mervyn King before Treasure Select Committee, November 25, 2008

${ }^{11}$ http://www.guardian.co.uk/business/2012/jul/13/tim-geithner-mervyn-king-libor
} 
emails, in which Geithner gave several suggestions to reform the LIBOR fixing process. These correspondences followed Barclays formulating worries in August 2007 about quotes of other panel banks. In October 2008 there has been an exchange between the deputy governor of the Bank of England, Paul Tucker, and Bob Diamond, the CEO of Barclays. Diamond's account of these exchanges have been interpreted by Barclays employees as suggestions to lower the LIBOR quotes. Tucker however rejects the idea that he meant Barclays to believe that it should quote lower. ${ }^{12}$ Barclays quotes were prior to October 2008 usually among the highest of the panel banks. This changed after October 2008, when the executive Jerry Del Missier ordered the head of the money market desk to lower the quotes. 13

The investigation of financial exposure as a driver of misquoting of banks has been pushed forward by Snider and Youle (2010). The first version of their paper is from autumn 2009. They find evidence for quote bunching around the pivotal quotes, the highest and lowest quotes which are included in the trimmed average. This is a clear indicator for strategic quoting of banks. The comparison between the Eurodollar and the LIBOR is also puzzling. Eurodollar are dollars which are held outside of US territory and thus outside of US jurisdiction. Comparing the Eurodollar bid rate and the LIBOR, which is an offer rate, we find prior to the start of the financial crisis in August 2007 a regular bid-ask spread. The bid rate was 6-12 basis points below the LIBOR quotes. This pattern changed in August 2007 and till summer 2011 the offer rate was below the ask rate. One may call this the Eurodollar-LIBOR paradox. The paradox is a clear indicator that the LIBOR quotes were not accurate but too low.

In May 2011 the Wall Street Journal wrote that regulators are focusing on Bank of America, Citibank and UBS in their investigation. 14 The LIBOR scandal however really broke lose when Barclays admitted misquoting in the LIBOR fixing and agreed to a settlement in June 2012. Barclays not only agreed to a fine of $\sim \$ 450$ million but also to the publishment of internal email correspondences. Some of these emails have become famous and can be now found on the web. ${ }^{15}$ One email reads: "Hi Guys, We got a big position in $3 \mathrm{~m}$ libor for the next 3 days. Can we

\footnotetext{
${ }^{12}$ http://www.guardian.co.uk/business/2012/jul/13/tim-geithner-mervyn-king-libor

${ }^{13} \overline{\text { http://www.guardian.co.uk/business/2012/jul/16/libor-barclays-fsa-jerry-del-missier }}$

${ }^{14} \overline{\text { http://online.wsj.com/article/SB10001424052748703818204576205991698548286.html }}$

15 Excerpts can be found here: http://www.telegraph.co.uk/finance/newsbysector/banksandfinance/9359392/Keyemails-how-Barclays-manipulated-Libor.html
} 
please keep the libor fixing at 5.39 for the next few days. It would really help. We do not want it to fix any higher than that. Tks a lot." This mail was sent on September 13, 2006, from a senior trader in New York to a LIBOR submitter. Another exchange is summarized in the Telegraph ${ }^{16}$ as follows: On 26 October 2006, an external trader made a request for a lower three month US dollar LIBOR submission. The external trader stated in an email to Trader G at Barclays "If it comes in unchanged I'm a dead man". Trader G responded that he would "have a chat". Barclays' submission on that day for the three month US dollar LIBOR was half a basis point lower than the day before, rather than being unchanged. The external trader thanked Trader G for Barclays' LIBOR submission later that day: "Dude. I owe you big time! Come over one day after work and I'm opening a bottle of Bollinger". A Bollinger is a champagne brand. Important to note is that there has been internal and external communication of the money market desk at Barclays, which is responsible for the LIBOR quote.

Besides Barclays, other banks that have admitted misquoting and settled with the regulators are UBS ( \$1.5 billion) and RBS ( \$600 million). Additionally to ongoing investigations by financial authorities against the LIBOR setting banks, LIBOR banks are also sued in civil court. Homeowners in the United States filed a class action against LIBOR banks in October 2012. Flexible mortgage rates which are tied to the LIBOR are mostly reset on the first day of a month. ${ }^{17}$ Statistical analysis has indicated that on this day quotes have gone up on a regular basis. This is a clear indicator that banks tried via LIBOR manipulation to make mortgage repayments artificially high. In April 2012 a number of municipalities in the United States filed a class action lawsuit against the LIBOR banks as due to the too low quotes the municipalities earned less on their interest rate swaps. The loss to the municipalities is quantified as being at least $\$ 6$ billion. 18

These civil court lawsuits and the investigations of the financial authorities are ongoing (This is written May 2013). More penalties against the banks and more precise estimations of the

\footnotetext{
${ }^{16}$ http://www.telegraph.co.uk/finance/newsbysector/banksandfinance/9359392/Key-emails-how-Barclaysmanipulated-Libor.html

'thttp://www.forbes.com/sites/halahtouryalai/2012/10/15/banks-rigged-libor-to-inflate-adjustable-ratemortgages-lawsuit/

${ }^{18}$ http://www.bloomberg.com/news/2012-10-09/rigged-libor-hits-states-localities-with-6-billion-municredit.html
} 
magnitude of the scandal are expected in the next months and years. The proof of fraud is not easy as the LIBOR quotes are supposed to be best guesses of the borrowing costs. They are not necessarily based on transactions and due to the illiquidity of the interbank market it is often not clear, what the true borrowing costs are. Moreover, there does not exist a transaction database for actual interbank lending. The case for such a database is made in Eisl et al. (2013) and Abrantes-Metz and Evans (2012a). The strategy of the prosecutors will mainly be a combination of finding whistleblowers, analyzing internal and external communication of the LIBOR setting employees in the banks, and statistical investigations of the quote patterns. In particular, the statistical investigations will consider quote clustering at the pivotal quantiles, the relation of LIBOR to other measures of borrowing rates, timing of movements of LIBOR quotes, and similarity of LIBOR quotes. There are indications that the manipulation of the LIBOR goes back to the very first days of its existence. Reports say that fraud dates back till at least $1991{ }^{19}$, other sources speak of fraud already in the late $80 \mathrm{~s}{ }^{20}$ In an updated version of their paper, Snider and Youle (2012) consider data up to June 2012. They are able to find indications that LIBOR manipulation has continued after the financial crisis till 2011, but their tests show no unregular patterns after August 2011.

\subsection{Policy responses to the LIBOR scandal}

The LIBOR scandal has led to an intense and ongoing debate on how to ensure that the LIBOR cannot be manipulated in the future and that it in fact represents the true borrowing costs on the unsecured interbank market. The discussion paper by Wheatley (2012b) and the Wheatley review by Wheatley (2012a) are the most important documents which summarize possible avenues for reforming the LIBOR. They were written by the CEO of the Financial Conduct Authority (FCA), Martin Wheatley. The FCA is the quasi-governmental regulating agency for financial firms in Britain. A summary of the Wheatley review is given by Garcia (2012) and criticism and alternative reform suggestions are put forward by Abrantes-Metz and Evans (2012b) and Abrantes-Metz and Evans (2012a). The following are the main suggestions given by the review. 1. Reduce the number of currencies and maturities. This facilitates the usage

\footnotetext{
${ }^{19}$ Mark Gongloff, LIBOR was happening in 1991, July 27, 2012, Huffington Post

${ }^{20}$ http://www.economist.com/node/21558281
} 
of actual transaction data. 2. Make the quotes of individual banks temporarily anonymous, the suggested timeframe is 3 months. This adresses possible reputation concerns and makes collusion of the banks harder. 3. Increase the panel sizes. This decreases the marginal influence of a single bank on the index. 4. Make a clear separation of the trading desk of a panel bank and the bank's LIBOR submitting quotes to give the submitters no indication from which quote patterns the bank would profit. 5. Change from the BBA to a different agency which administers the LIBOR. Some banks are members of the BBA, thus had to oversee themselves. The responsible agency should be independent. All reform suggestion put forward by Wheatley will be implemented by the Treasury.

While all these suggestions are sensible and will be supported by the results of our theoretical model in the following sections, it is important to note that the main idea of asking the banks what their perceived borrowing costs are is not changed in the Wheatley review. The reasoning for sticking qualitatively to the existing mechanism is on the one hand the lack of alternative benchmarks and on the other hand the fact that the LIBOR is currently included in the contracts and the legal way of phasing out the LIBOR is complicated. Thus, according to Wheatley, for reasons of the stability of the financial markets, it is important to continue with the LIBOR. These arguments are not wrong. However, and here we begin to speculate about the future of the LIBOR, we expect that the market will move away from using the LIBOR. Alternative benchmarks will be developed and used. The LIBOR is an anachronism from a time when the LIBOR was only used to find out the interbank offered rate but when this rate was not used for other purposes like fixing derivatives. Interesting in this context is also research by psychologists, like Gino et al. (2009). They show that once a culture of fraud has begun to establish itself, it is very difficult to reestablish a culture of trust.

\section{The model}

Let $N=\{1, \ldots, n\}$ be the set of players playing the game, i.e. panel banks. Nature chooses the state $\theta \in \Theta$, where $\Theta=\mathbb{R}_{+}^{n}$. All players are informed about the state of the world, i.e. all

\footnotetext{
${ }^{21}$ http://www.moneymarketing.co.uk/regulation/treasury-to-implement-all-wheatleys-liborrecommendations/1059869.article
} 
players know the realization of the $n$-dimensional random variable $\theta$. The strategy of player $i$ is a function $s_{i}: \Theta_{i} \rightarrow X_{i}$, mapping states of nature into the set $X_{i}$ of quotes of player $i$, where $X_{i}=\mathbb{R}_{+}$. We assume $X_{i}$ to be the same for all players. To complete the definition of a strategic game, we suggest the following utility function.

$$
u_{i}\left(x_{i}, x_{-i}, \theta_{i}\right)=\nu_{i} I\left(x_{i}, x_{-i}\right)-\left(x_{i}-\theta_{i}\right)^{2}+\lambda_{i}\left(I\left(x_{i}, x_{-i}\right)-x_{i}\right)
$$

The particular form of this utility function needs some explanation. $I(x)$ denotes a statistic, which depends on all quotes of all players. The games which we investigate will be completely identical except for the fact that we will consider different kind of statistics like the average or the median. $\nu_{i}$ gives the financial exposure of bank $i$ to the index and the utility is linear in this component. Players face quadratic costs for deviation from their true personal costs. The third component describes reputational concerns of the players of their own quotes relative to the index and play a role as all individual quotes are published in addition to the index. A high quote relative to the index may indicate weakness of the bank. The banks weigh their reputational concerns with the real number $\lambda_{i}$.

The linear quadratic structure is a simplification of the true incentive structure that may entail misrepresentation of the banks. However, in the very few other theoretical contributions that investigate the LIBOR problem, namely Chen (2012) and Snider and Youle (2012), similar utility functions are used. A reasoning behind the justification of the structure besides simplicity and tractability goes along the following lines: The more extreme the misrepresentation, the more likely a detection of it and the penalty payment is relative to the magnitude of the misrepresentation. This gives a quadratic penalty term. For small deviations approximations with linear functions are possible, and a huge number of derivatives based on the LIBOR have linear or at least partially linear payoffs. We note moreover that our primary goal of this paper is to come to qualitative, not quantitative, conclusions concerning the vulnerability of the LIBOR and related mechanism. We aim to provide ways how to think about the LIBOR problem that may add to the understanding of how to redesign the mechanism in a sensible and more robust way.

In addition to the $n$ players, there is a mechanism designer concerned about the index. This designer does not know the true state of the world $\theta$, but only gets to know the quotes $x$ and is 
only allowed to use the vector of quotes to determine the index. The objective of the designer is to ensure $I(\theta)=I(x)$, in the ideal case. In general, the designer wants to design the game and the statistic such that the statistic gives, loosely speaking, a good sense of the private values of the players. This is in line with the description of the LIBOR by the BBA: 'LIBOR reflects the rates at which contributor banks can borrow money from each other each day, in the world's ten major currencies and for 15 borrowing periods ranging from overnight to 12 months. 422

\section{Equilibrium analysis of the games}

We now investigate different games. The games are completely identical except for the statistic in the payoff functions of the players. We begin with the easiest case, the average, before we analyze more complicated statistics like the median or averages which are trimmed in different kind of ways. In the average case all quotes directly influence the index. However, in the other two games, the Median Game and the Trimmed Average Game, misquoting may change the index even if the quote does not directly enter the calculation. Misrepresentation may alter the order of the quotes. For a simple example, see figure 3 in the appendix.

\subsection{The benchmark: The Average Game}

In this subsection we set $I(x)=A(x)=\sum_{i=1}^{n} \frac{x_{i}}{n}$, i.e. all quotes influence directly the statistic which is the average of all quotes submitted. We do not impose any restrictions concerning the $\lambda_{i}$. Thus, the utility function of an individual bank $i$ is

$$
u_{i}\left(x_{i}, x_{-i}, \theta_{i}\right)=\nu_{i} A\left(x_{i}, x_{-i}\right)-\left(x_{i}-\theta_{i}\right)^{2}+\lambda_{i}\left(A\left(x_{i}, x_{-i}\right)-x_{i}\right) .
$$

We call the defined game the Average Game which will serve as a benchmark. As all quotes of all banks always enter the calculation of the average, the banks individually optimize without any strategic considerations. The individual optimization is independent of the optimization of all competing players, thus the Average Game is in a sense not really a strategic game. The

\footnotetext{
${ }^{22}$ http://www.bbalibor.com/bbalibor-explained/faqs
} 
first order condition (FOC) is

$$
\text { FOC }: \frac{\nu_{i}}{n}-2 x_{i}+2 \theta_{i}+\frac{\lambda_{i}}{n}-\lambda_{i}=0 .
$$

This gives us our first theorem and two corollaries which immediately follow.

Theorem 1. The bank's optimal quote is $x_{i}=\theta_{i}+\frac{\nu_{i}}{2 n}+\frac{\lambda_{i}}{2 n}-\frac{\lambda_{i}}{2}$. The deviation in the quotation $x_{i}$ from the true costs $\theta_{i}$ is $\frac{\nu_{i}}{2 n}+\frac{\lambda_{i}}{2 n}-\frac{\lambda_{i}}{2}$. In particular, the quotes decrease in $\lambda_{i}$ and $A(x)=$ $\sum_{i=1}^{n} \theta_{i}+\frac{\nu_{i}}{2 n}+\frac{\lambda_{i}}{2 n}-\frac{\lambda_{i}}{2}$.

Corollary 2. Incentive compatibility can be guaranteed via a Groves-scheme with transfers $t_{i}=-\left(\frac{\nu_{i}}{2 n}+\frac{\lambda_{i}}{2 n}-\frac{\lambda_{i}}{2}\right)$.

Corollary 3. If reputational concerns do not play a role $\left(\lambda_{i}=0\right)$, the misrepresentation decreases in the number $n$ of banks in the panel and $q_{i}=\theta_{i}+\frac{\nu_{i}}{2 n} \rightarrow \theta_{i}$ for $n \rightarrow \infty$.

These findings follow the intuition. The higher the individual concern about the own quote's relation to the average, the more important becomes the reputational concern component in the utility function of the banks. This unilaterally leads to lower quotes, as noted in theorem 1 . As we can calculate the precise misrepresentation of the individual player in the Average Game, we can guarantee truthful quotations of the players with a Groves-scheme like in Groves (1973). Transfer payments are chosen such that misrepresentation incentives are exactly reserved and truthful quoting becomes an equilibrium. The third finding, corollary 3 , gives information when the often discussed approach of an increase in the panel sizes may help to mitigate the misrepresentation problem. The next two results, theorem 4 and corollary 5 , continue the investigation of this approach.

The society which is represented by the mechanism designer is concerned with the question whether banks quote their true costs and more importantly, if $A(x)=A(\theta)$. The following theorem and the corollary address this problem.

Theorem 4. An individual bank quotes honestly if and only if $\nu_{i}=\lambda_{i}(n-1)$. The average gives the actual interbank borrowing rate if and only if $\sum_{i=1}^{n} \nu_{i}=\sum_{i=1}^{n} \lambda_{i}(n-1)$.

The following observation is an immediate consequence of the theorem. 
Corollary 5. Increasing the number $n$ of players solves the misrepresentation problem in the limit if and only if $\lambda_{i}=0$ for all $i$.

For completion, we now present the straightforward proof of theorem 4 .

Proof.

$$
\begin{gathered}
A(x)=\frac{\sum_{i=1}^{n} \theta_{i}+\frac{1}{2 n} \sum_{i=1}^{n} \nu_{i}+\sum_{i=1}^{n} \frac{\lambda_{i}}{2 n}-\sum_{i=1}^{n} \frac{\lambda_{i}}{2}}{n}=\frac{\sum_{i=1}^{n} \theta_{i}}{n} \\
\Leftrightarrow \frac{1}{2 n} \sum_{i=1}^{n} \nu_{i}+\sum_{i=1} n \frac{\lambda_{i}}{2 n}-\sum_{i=1}^{n} \frac{\lambda_{i}}{2}=0 \\
\Leftrightarrow \sum_{i=1}^{n} \nu_{i}=\left(\frac{\sum_{i=1}^{n} \lambda_{i}}{2}-\frac{\sum_{i=1}^{n} \lambda_{i}}{2 n}\right) 2 n=\sum_{i=1}^{n} \lambda_{i}(n-1) .
\end{gathered}
$$

As panel banks also trade with non panel banks and with other entities like hedge funds, the condition $\sum_{i=1}^{n} \nu_{i}=\sum_{i=1}^{n} \lambda_{i}(n-1)$ is normally not satisfied even in the case $\lambda_{i}=0$ for all $i$. The default outcome of the average index is such that $A(\theta) \neq A(x)$, thus the average index is an index which normally does not accurately reflect the true borrowing costs on the interbank market. However, as theorem 1 shows, increasing the number of banks can attenuate the misrepresentation problem if $\lambda$ is the null vector. Then there is no systemic misquoting in one direction. This is a difference to the case $\lambda_{i}>0$ for some $i \in N$.

In the case of a nonnull vector $\lambda$, the more banks there are in the panel, the less likely it is that the average of all quotes is the average of all private costs. This makes sense with the following intuition. The more banks there are in the panel, the smaller the marginal impact of a single bank, the more extreme the financial exposure has to be in the positive direction to counterbalance the reputational concerns.

Before we turn to the Median Game in the next subsection, let us briefly evaluate our findings. We have shown a number of interesting results. For example, we can give an explanation why deviations of the LIBOR from market based indicators have been observed since the financial crisis has unfurled. In times of financial crisis, the likelihood of bank runs is higher and reputational concerns of banks become more important. This is reflected in the utility function of the banks by a higher $\lambda_{i}$. In calm markets, which may correspond to the $\lambda_{i}=0$ for all $i$ 
case, banks were primarily concerned with their financial exposure to the LIBOR and not with reputation. Some banks have positive exposure, some negative exposure, the overall effect may cancel out. If there is market turmoil like in a financial crisis, there is an additional incentive for the banks to misrepresent which unanimously points in one direction, in the direction of lower quoting. Exactly this can be observed since the crisis of $2007 / 2008$ unfurled. The LIBOR left the traditional close connection to market indicators like credit default swaps (CDS) and has been much lower in the following months. To get these results, we made simplifications. Most importantly, we calculated the index as an average and not as a trimmed average. This leads to the fact that banks do not have to reason about the quoting pattern of the other banks. We now turn to a true game theoretic model and investigate the Median Game, where the statistic in the utility function of the players is the median of all submitted quotes.

\subsection{The Median Game}

The current design of the LIBOR as an average of quotes between two thresholds given by the $25 \%$ and $75 \%$ quantiles leads us to the formulation and analysis of what we call the Median Game. Consider now the model setup of section 3 with the statistic $I(x)=M(x)$, where $M(x)$ gives the median of the vector of quotes $x$. We have to distinguish between even and uneven numbers of players and consider the order statistic $\left(x^{(1)}, \ldots, x^{(n)}\right)$, i.e. $x^{(1)} \leq \ldots \leq x^{(n)}$. Suppose $n$ is uneven. Then $M(x)=x^{\left(\frac{n+1}{2}\right)}$. For even $n$, the median is the average of the two middle quotes, $M(x)=\frac{1}{2} x^{\left(\frac{n}{2}\right)}+\frac{1}{2} x^{\left(\frac{n+2}{2}\right)}$. From now on, we set $\lambda_{i}=0$ for all $1 \leq i \leq n$. The utility function in the Median Game has then the following form

$$
u_{i}\left(x_{i}, x_{-i}, \theta_{i}\right)=\nu_{i} M\left(x_{i}, x_{-i}\right)-\left(x_{i}-\theta_{i}\right)^{2} .
$$

We investigate separately the Median Game for an uneven number of players and an even number of players and start with the uneven case. An example for $n=3$ is given in figure 1 in the appendix. The following are two important observations which simplify the further analysis enormously.

Theorem 6. Suppose the number of players in the Median Game is uneven. In every Nash equilibrium it holds $x_{i}=\theta_{i}$ or $x_{i}=M(x)$. 
Proof. We prove this by showing that all other strategies are strictly dominated. Suppose $x_{i} \neq \theta_{i}$ and $x_{i} \neq M(x)$. In a sufficiently small environment of $x_{i}$ there exists $\tilde{x}_{i}$ such that $M\left(\tilde{x}_{i}, x_{-i}\right)=M(x)$ and $\left(\theta_{i}-\tilde{x}_{i}\right)^{2}<\left(\theta_{i}-x_{i}\right)^{2}$. Thus the strategy $\tilde{x}_{i}$ strictly dominates $x_{i}$ which implies that $x_{i}$ is not played in any Nash equilibrium.

Corollary 7. Suppose the number of players in the Median Game is uneven and $n \geq 3$. In Nash equilibrium at least one player quotes truthfully.

Proof. The only thing we have to show with reference to theorem 6 is that $\theta_{i} \neq x_{i}=M(x)$ for all $i$ cannot be a Nash equilibrium. But this is immediate, as any player can profitably deviate to truth telling. The median does not change but the penalty decreases.

We now turn to the uneven case, for which an analogue to theorem 6 exists while the corollary is not true as we show by means of an example.

Theorem 8. Suppose the number of players in the Median Game is even. In every Nash equilibrium it holds $x_{i}=\theta_{i}$ or $x_{i} \in\left\{x^{\left(\frac{n}{2}\right)}, x^{\left(\frac{n+2}{2}\right)}\right\}$

Proof. The proof is almost a copy of the proof of theorem 6. We show that all other strategies are strictly dominated. Suppose $x_{i} \neq \theta_{i}$ and $x_{i} \notin\left\{x^{\left(\frac{n}{2}\right)}, x^{\left(\frac{n+2}{2}\right)}\right\}$. In a sufficiently small environment of $x_{i}$ there exists $\tilde{x}_{i}$ such that $M\left(\tilde{x}_{i}, x_{-i}\right)=M(x)$ and $\left(\theta_{i}-\tilde{x}_{i}\right)^{2}<\left(\theta_{i}-x_{i}\right)^{2}$. Thus the strategy $\tilde{x}_{i}$ strictly dominates $x_{i}$ which implies that $x_{i}$ is not played in any Nash equilibrium.

Comment 1. Suppose the number of players in the Median Game is even. There exist equilibria in which no player answers truthfully.

Proof. The proof is given by means of an example. For $n=2$ we are back in the Average Game and players always misrepresent for financial exposure different from 0 . Thus consider $n=4$. The constellation for the example has to be such that two players have negative financial exposure and the other two have positive financial exposure. The negative financial exposure players have higher private costs than the positive financial exposure types. Then there exist parameter constellations in which the low private value types quote higher than the high private value types such that all misrepresent and no single player can unanimously deviate profitably. 
One parameter constellation that works is the following. $\theta_{1}=\theta_{2}=1.4, \nu_{1}=\nu_{2}=1, x_{1}=x_{2}=$ 1.6, and $\theta_{3}=\theta_{4}=1.6, \nu_{3}=\nu_{4}=-1, x_{3}=x_{4}=1.4$. For $n>4$ add identical numbers of identical players to the high and low type, respectively.

These findings facilitate the further characterization of Nash equilibria in the Median Game. In the next section we consider the case $n=3$ and characterize the equilibria for the heterogenous private value, the heterogenous financial exposure, and the general case.

\subsection{The three player Median Game}

\subsubsection{Heterogenous costs}

In this subsection, we assume $n=3$ and that all players have exactly the same financial exposure $\nu_{i}=\nu_{j}=\nu_{k}=\nu$ to the median index. We set $\nu \geq 0$, the case $\nu \leq 0$ is solved similarly. The private values of the players are however possibly different. We investigate the four arising cases: $1 . \theta_{i}=\theta_{j}=\theta_{k}, 2 . \theta_{i}=\theta_{j}<\theta_{k}, 3 . \theta_{i}<\theta_{j}=\theta_{k}$ and $4 . \theta_{i}<\theta_{j}<\theta_{k}$. We start with the easiest case, identical values for all three players.

1. $\theta_{i}=\theta_{j}=\theta_{k}=\theta$ :

In the case of identical costs for all agents, there arise exactly two different kind of equilibria; the truth telling equilibrium and an equilibrium in which one player quotes honestly and the other two quote the same, but not the truth.

Theorem 9. If $\theta_{i}=\theta_{j}=\theta_{k}=\theta$, then for any equilibrium it holds that either $M(x)=x_{l}=\theta$ for all $l=i, j, k$ or $\exists l \in\{i, j, k\}$ such that $\theta=x_{l}$ and $M(x)=x_{m}=x_{n} \neq \theta$ for $m, n \neq l$. In the second type of equilibrium the dishonest players quote at most $M(x)=x_{m}=\frac{\nu}{2}+\theta$.

Proof. If all players quote honestly, there is no possibility to unilaterally deviate profitably. Deviation in the other equilibrium is not profitable for the honest player $i$ as the median would not change and the penalty for lying is already minimal. For the other two players $j, k$ a deviation is not profitable if their quote $x_{j}$ is between $\theta$ and the maximizer of $\nu x_{j}-\left(x_{j}-\theta\right)^{2}$, thus if $x_{j} \leq \frac{\nu}{2}+\theta$. If $x_{j}>\frac{\nu}{2}+\theta$, then the deviation to $x_{j}=\frac{\nu}{2}+\theta$ were profitable. Thus both 
type of quotation patterns constitute Nash equilibria. It remains to show that these are all. Suppose $x_{i}<x_{j}<x_{k}$. Then either $i$ or $k$ can profitably deviate to quoting $x_{j}$.

$2 . \theta_{i}<\theta_{j}=\theta_{k}:$

The same two types of equilibria arise as in the identical costs case.

Theorem 10. If $\theta_{i}<\theta_{j}=\theta_{k}$, then in any equilibrium it holds that either $x_{i}=\theta_{i}$ and $M(x)=x_{j}=x_{k}=\theta_{j}$ or there exist players $m, n \in\{i, j, k\}$ such that $M(x)=x_{m}=x_{n}>\theta_{k}$ and $x_{l}=\theta_{l}$. In the second type of equilibrium, player $i$ quotes at most $M(x)=x_{i}=\frac{\nu}{2}+\theta_{i}$ while the other two quote at most $x_{j}=\frac{\nu}{2}+\theta_{j}$. It holds for the nontruthful quote $x_{j}>\theta_{j}$. In particular, if $\frac{\nu}{2}+\theta_{i}<\theta_{j}$, player $i$ quotes always truthfully.

Proof. The truth equilibrium exists as there is no possibility to unilaterally deviate profitably. In the other equilibrium the two players who quote the same control the median. As the truthful quote is below the quote of the two equal quoting players, one of the two equal quoting players can only change the median by deviating to a lower quote, which is in the specified intervals not profitable. Higher quotation would only induce a higher penalty.

3. $\theta_{i}=\theta_{j}<\theta_{k}:$

In this situation, the truth equilibrium ceases to exist. Moreover, depending on the true cost structure, new types of equilibria may appear.

Theorem 11. If $\frac{\nu}{2}+\theta_{i}<\theta_{k}$, there exists only the equilibrium in which $i$ or $j$ quotes the truth, $k$ quotes the truth and the other $i$ or $j$ sets the median by quoting $M(x)=x_{i}=\theta_{i}+\frac{\nu}{2}$. If $\frac{\nu}{2}+\theta_{i} \geq \theta_{k}$, there exists only the equilibrium in which one player quotes the truth and the other two players quote $M(x)=x_{l} \in\left[\theta_{k}, \frac{\nu}{2}+\theta_{i}\right]$.

Proof. The truth equilibrium does not exist as $i$ and $j$ could both profitably deviate by quoting higher. If $\frac{\nu}{2}+\theta_{i}<\theta_{k}$, the nontruthful player sets the median by its optimal quote $x_{i}=\theta_{i}+\frac{\nu}{2}$. If $\frac{\nu}{2}+\theta_{i} \geq \theta_{k}$, the deviation of the nontruthful player falls in the domain where the high cost player also can deviate. By quoting the same and thereby controlling the median, no individual player would like to deviate. The low quoter cannot affect the median, and the higher quoters quote in the area where a deviation to a lower quote would not be advantageous. 
$4 . \theta_{i}<\theta_{j}<\theta_{k}:$

In the case where all players face different individual costs, the equilibrium structure is similar to case 3 .

Theorem 12. If $\theta_{j}+\frac{\nu}{2} \leq \theta_{k}$, then there only exists the equilibrium in which $\theta_{i}=x_{i}, \theta_{k}=x_{k}$ and $M(x)=x_{j}=\theta_{j}+\frac{\nu}{2}$. If $\theta_{j}+\frac{\nu}{2}>\theta_{k}$, then there exists the equilibrium in which $\theta_{i}=x_{i}$ and $M(x)=x_{j}=x_{k} \in\left[\theta_{k}, \theta_{j}+\frac{\nu}{2}\right]$. If additionally $\theta_{i}+\frac{\nu}{2} \geq \theta_{k}$ then there exists an equilibrium, in which players $i$ and $j$ or $k$ quote the same $M(x)=x_{i} \in\left[\theta_{k}, \theta_{i}+\frac{\nu}{2}\right]$ and the other player $j$ or $k$ quotes the truth.

Proof. The truth equilibrium does not exist as the median cost player can always deviate profitably. The range of possible deviations from the truth depends on the underlying cost structure. With similar reasoning as in the proof of theorem 11 one proves that the given quote patterns in fact represent equilibria.

These four theorems completely characterize the set of pure Nash equilibria in the Median game in the three player cases, where players have identical financial exposure but heterogenous costs. Depending on the cost constellation, three different types of equilibria arise. The truthtelling equilibrium, an equilibrium where two players control the median and the third quotes truthfully, and an equilibrium in which the top and bottom cost players quote truthfully while the middle cost player deviates in a maximal sense. Interestingly, the second and the third equilibrium type are mutually exclusive. Moreover, the equilibrium set in the second type of equilibrium is indeterminate.

Indeterminacy of equilibrium sets is also a result we get in the next subsection, in which we consider heterogenous exposure but identical private values.

\subsubsection{Heterogenous exposure}

In this subsection, we continue to assume $n=3$ and $\theta_{i}=\theta_{j}=\theta_{k}=\theta \geq 0$. However, we now allow heterogeneity concerning the financial exposure of the players. We investigate the four different cases: $1.0 \leq \nu_{i} \leq \nu_{j} \leq \nu_{k}, 2 . \nu_{i} \leq 0 \leq \nu_{j} \leq \nu_{k}, 3 . \nu_{i} \leq \nu_{j} \leq 0 \leq \nu_{k}$ and 4 . 
$\nu_{i} \leq \nu_{j} \leq \nu_{k} \leq 0$. Cases 2. and 3. are the interesting cases, the cases 1 . and 4 . with exclusively positive or negative financial exposure are similar to the results of the preceding subsection. Basically one has to add indices to the financial exposure. We summarize the findings for the four different cases of financial exposure to the median in the following theorem which characterizes the equilibria. Case 1 . corresponds to - case 4 . and case 2. to -case 3.

Theorem 13. 1.If $\theta_{i}=\theta_{j}=\theta_{k}=\theta$ and $0 \leq \nu_{i} \leq \nu_{j} \leq \nu_{k}$, the only equilibria are the truth telling equilibrium and the equilibrium in which one player quotes truthfully and two players lie. In the second type of equilibrium the dishonest players $m, n$ quote $M(x)=x \in$ $\min _{s \in\{m, n\}}\left[\theta, \frac{\nu_{s}}{2}+\theta\right]$.

2. If $\theta_{i}=\theta_{j}=\theta_{k}=\theta$ and $\nu_{i} \leq 0 \leq \nu_{j} \leq \nu_{k}$, the only equilibria are the truth telling equilibrium and the equilibrium in which $i$ quotes the truth $x_{i}=\theta$ and $j, k$ quote $M(x)=x_{j}=x_{k} \in\left[\theta, \frac{\nu_{j}}{2}+\theta\right]$. 3. If $\theta_{i}=\theta_{j}=\theta_{k}=\theta$ and $\nu_{i} \leq \nu_{j} \leq 0 \leq \nu_{k}$, the only equilibria are the truth telling equilibrium and the equilibrium in which $k$ quotes the truth $x_{k}=\theta$ and $j, k$ quote $M(x)=x_{j}=x_{k} \in$ $\left[\frac{\nu_{j}}{2}+\theta, \theta\right]$

4. If $\theta_{i}=\theta_{j}=\theta_{k}=\theta$ and $\nu_{i} \leq \nu_{j} \leq \nu_{k} \leq 0$, the only equilibria are the truth telling equilibrium and the equilibrium in which one player quotes truthfully and two players lie. In the second type of equilibrium the dishonest players $m, n$ quote $M(x)=x \in \max _{s \in\{m, n\}}\left[\frac{\nu_{s}}{2}+\theta, \theta\right]$.

Proof. As all players face the same costs and an individual cannot unilaterally change the index when all three players quote the same, truth is in all four cases an equilibrium.

1. and 4.: The maximal misrepresentation of an individual agent $l$ is $\frac{\nu_{l}}{2}$, if the exposure to the median is positive for all agents. This explains the minimum in 1 . The maximal misrepresentation of an individual agent $l$ in the negative exposure case is $\frac{\nu_{l}}{2}$. This leads to the maximum in 4 .

2. and 3.: The direction of possible misrepresentation of two agents is the same if and only if the financial exposure has the same sign. Thus, only players who both have positive or negative exposure can misrepresent in the same direction in equilibrium. The interval of possible equilibrium misrepresentations is determined by the player with the smaller absolute exposure which is player $j$.

Thus, we have, as theorem 9 suggested, only two types of equilibria: The truth equilibrium and 
the indeterminate set of equilibria. We now turn to the general characterization of the equilibria in the Median Game.

\subsubsection{General case}

In this subsection, we characterize the equilibria in the Median Game in the general case. We thus allow for heterogenous exposure and heterogenous costs. A case distinction is now made with respect to the quotes and not with respect to the private values and financial exposure as in the preceding subsections. Decisive in the proof will be to compare the regions of possible misrepresentations of agents. Formally, the interval of possible misrepresentations $I_{i}$ of player $i$ is the region in which player $i$ may quote in equilibrium. Equivalently, one may call it interval of possible quotes. It depends on the exposure $\nu_{i}$ and the $\operatorname{costs} c_{i}$. The following lemma characterizes it.

Lemma 4.1. The interval of possible misrepresentations $I_{i}$ is given by $I_{i}=\left[\theta_{i}, \theta_{i}+\frac{\nu_{i}}{2}\right]$

Proof. Suppose $\nu_{i} \geq 0$. Then player $i$ will always quote at least $\theta_{i}$ and not more than $\theta_{i}+\frac{\nu_{i}}{2}$ as for higher quotes the quadratic detection costs always dominate financial exposure benefits by misquoting. The $\nu_{i} \leq 0$ case is proved similarly.

The decisive trick for the complete characterization of the equilibria in the Median Game is to investigate all cases of possible overlappings between the intervals of possible misrepresentations.

Theorem 14. Suppose $\theta_{1} \leq \theta_{2} \leq \theta_{3}$.

(i) If $M(x)=x_{1}=x_{2}=x_{3}$, then $x_{1}=\theta_{1}=\theta_{2}=\theta_{3}$.

(ii) Suppose $x_{i}<M(x)=x_{j}<x_{k}$. Then $\operatorname{int}\left(I_{i}\right) \not \supset x_{j} \notin \operatorname{int}\left(I_{k}\right), x_{j}=\theta_{j}+\frac{\nu_{j}}{2}$ and $x_{i}=\theta_{i}$, $x_{k}=\theta_{k}$.

(iii) Suppose $M(x)=x_{i}=x_{j} \neq x_{k}$. Then $x_{k}=\theta_{k}$. If $\operatorname{sign}\left(\nu_{i}\right)=\operatorname{sign}\left(\nu_{j}\right)$, then $x_{i} \in I_{i} \cap I_{j}$. If $\nu_{i}<0<\nu_{j}$, then $i$ or $j$ quotes truthfully and the other player misrepresents in a maximal way. 
Proof. (i) Suppose all quotes are the same. Then everybody quotes truthfully as otherwise there would be a profitable deviation to truthtelling for the nontruthful player.

(ii) Suppose all quotes are different in equilibrium. Then the top and bottom quoter are truthful, else they would deviate in the direction of truthtelling. Moreover, the middle quoter misquotes maximally, else an $\epsilon$ change in the quote in the direction of financial exposure would be a profitable deviation. The middle quote cannot be in the interior of the maximal misrepresentation intervals of the two honest quoters as otherwise they would have a profitable deviation.

(iii) It holds $x_{k}=\theta_{k}$ as $k$ cannot influence the index.

If $\nu_{i} \geq 0 \leq \nu_{j}$ and $x_{k} \leq \min \left\{\theta_{i}, \theta_{j}\right\}$, any element of the intersection $I_{i} \cap I_{j}$ can be an equilibrium if both $i$ and $j$ quote it. If $x_{k} \geq \min \left\{\theta_{i}+\frac{\nu_{i}}{2}, \theta_{j}+\frac{\nu_{j}}{2}\right\}$ but $x_{k} \leq \theta_{i}+\frac{\nu_{i}}{2}$ or $x_{k} \leq \theta_{j}+\frac{\nu_{j}}{2}$, then the respective $i$ or $j$ would deviate to $x_{k}$, contradicting the assumption that $x_{i}=x_{j}$ as for the other player $x_{k}$ lies outside of the interval of possible misrepresentations. Moreover, $x_{i}=\theta_{i}+\frac{\nu_{i}}{2}=\theta_{j}+\frac{\nu_{j}}{2}$. If $x_{k} \in I_{i} \cap I_{j}$, then $x_{i}=x_{j}>x_{k}$.

If $\nu_{i}<0<\nu_{j}$, it cannot be that in equilibrium $x_{i}=x_{j} \neq x_{k}$ and both $i$ and $j$ lie. One player, say $i$, would have a profitable deviation in the direction of private costs which does not change the median. Thus, assume wlog that player $i$ quotes truthfully. Player $j$ misrepresents maximally, $x_{i}=\theta_{i}=\theta_{j}+\frac{\nu_{j}}{2}$. Otherwise, $j$ would have a profitable deviation.

Important to note is that we do not get qualitatively new equilibria in comparison to the preceding subsections. We do not explicitly show existence of the equilibria in theorem 14 but we investigate all possible quote patterns of three players and which of these may qualify as equilibria. We again have three possible types of equilibria, the truthful equilibrium, the maximal misrepresentation of the middle quoter and truthful quotes of the top and bottom quoter, and the possibly indeterminate equilibrium type where two players quote the same and the third quotes truthfully. Examples of parameter constellations of the $\theta$ and $\nu$ vectors that lead to the existence of the respective equilibrium types have been given in the previous subsections 4.3 .1 and 4.3 .2 . 


\subsection{The Trimmed Average Game}

In the preceding subsection we investigated the Median Game and in particular provided a characterization of the equilibrium set in the three player case. The LIBOR is at the moment calculated as a trimmed average and not as a median or average. To discriminate between these three statistics, it is necessary to consider $n \geq 5$. For $n=1$ and $n=2$ all notions coincide. For $n=3$ the trimmed average can be either defined as average of all quotes or as the middle quote which is the median. Similarly for $n=4$. The average of the two middle quotes is the median. However, $n=5$ is the smallest number for which these three statistics are distinct. As utility function we take as before $u_{i}\left(x_{i}, x_{-i}, \theta_{i}\right)=\nu_{i} M(x)-\left(x_{i}-\theta_{i}\right)^{2}$ in the Median Game, $u_{i}\left(x_{i}, x_{-i}, \theta_{i}\right)=\nu_{i} A(x)-\left(x_{i}-\theta_{i}\right)^{2}$ in the Average Game, and $u_{i}\left(x_{i}, x_{-i}, \theta_{i}\right)=\nu_{i} L(x)-\left(x_{i}-\theta_{i}\right)^{2}$ in the Trimmed Average Game. The statistic in the Trimmed Average Game is defined as $L(x)=\sum_{i=2}^{4} \frac{x^{(i)}}{3}$ in the $n=5$ player game, where $x^{(i)}$ denotes the order statistic. We call this statistic $L(x)$ as this is closest to the original LIBOR calculation rule. See figure 2 in the appendix for the actual rule for $6 \leq n \leq 18$.

We start by presenting general findings for the Trimmed Average Game. They are close to the findings for the Median Game, in particular to the even case. Denote the smallest quote and the highest quote which are averaged in the trimmed average by $x^{(l)}$ and $x^{(u)}$, respectively.

Theorem 15. Suppose $n \geq 4$ is the number of players in the Trimmed Average Game. Then in every Nash equilibrium it holds $x_{i}=\theta_{i}$ or $x^{(l)} \leq x_{i} \leq x^{(u)}$.

Proof. With the obvious notation changes, the proof is an exact copy of the proof of theorem 8.

Comment 2. Suppose $n \geq 4$. Then there exist equilibria in which all players misrepresent.

Proof. This is shown by means of an example. For $n=4$, the trimmed average and median coincide and the example of remark 1 gives the result. For even $n$ this example for the median also works for the trimmed average if we add identical high and low value players. For uneven $n \geq 5$ consider the example for $n-1$ and add one identical high or low value player. This completes the proof of remark 2 . 
The next theorem formulates a sufficient criterion for misrepresentation in equilibrium.

Theorem 16. If $\theta_{i} \in\left(x^{(l)}, x^{(u)}\right)$, then $\theta_{i} \neq x_{i}$ if $\nu_{i} \neq 0$.

Proof. Assume $\nu_{i}<0$. Suppose bank $i$ quotes $x_{i}=\theta_{i} \in\left(x^{(l)}, x^{(u)}\right)$. This quote gives utility $u_{i}\left(x_{i}, x_{-i}, \theta_{i}\right)=\nu_{i} L\left(\theta_{i}, x_{-i}\right)$. Consider the quote $\tilde{x}_{i}=\theta_{i}-\epsilon$ for $\epsilon>0$ sufficiently small such that $\tilde{x}_{i} \in\left(x^{(l)}, x^{(u)}\right)$. This quote gives utility $u_{i}\left(\tilde{x}_{i}, x_{-i}, \theta_{i}\right)=\nu_{i} L\left(\tilde{x}_{i}, x_{-i}\right)-\left(\tilde{x}_{i}-\theta_{i}\right)^{2}$. Comparing the two utilities, it holds $\nu_{i} L\left(\tilde{x}_{i}, x_{-i}\right)>\nu_{i} L\left(\theta_{i}, x_{-i}\right)$ and $-\left(\tilde{x}_{i}-\theta_{i}\right)^{2}<0$. As the first inequality depends linearly on the quote and the second quadratically, for sufficiently small $\epsilon$ it holds $\nu_{i} L\left(\tilde{x}_{i}, x_{-i}\right)-\nu_{i} L\left(\theta_{i}, x_{-i}\right)-\left(\tilde{x}_{i}-\theta_{i}\right)^{2}>0$. Thus, we have found a strategy which strictly dominates $x_{i}=\theta_{i}$. The case $\nu_{i}>0$ is proven analogously.

For sufficiently large exposure to the index a bank will quote $x^{(l)}$ or $x^{(u)}$, depending on the sign of the exposure.

Theorem 17. For sufficiently large exposure $\left|\nu_{i}\right|$ to the LIBOR a bank will always quote $x^{(l)}$ if $\nu_{i}<0$ and $\theta_{i} \geq x^{(l)}$. Similarly, if $\nu_{i}>0$ and $\theta_{i} \geq x^{(u)}$, a bank will always quote $x^{(u)}$ provided sufficiently large exposure to the LIBOR.

Proof. Suppose $\nu_{i}<0$ and $\theta_{i} \geq x^{(l)}$. Quoting $x^{(l)}$ gives the utility $u\left(x^{(l)}, x_{-i}, \theta_{i}\right)=\nu_{i} L\left(x^{(l)}, x_{-i}\right)-$ $\left(x^{(l)}-\theta_{i}\right)^{2}$. Thus, whether quoting $x^{(l)}$ strictly dominates any other quote $\hat{x}_{i}>x^{(l)}$ depends on the relation of the first and the second term in the utility function. For sufficiently large $\left|\nu_{i}\right|$ quoting $x^{(l)}$ strictly dominates any other quote. The case $\nu_{i}>0$ and $\theta_{i} \leq x^{(u)}$ is proven analogously.

Comment 3. Snider and Youle (2010) were the first to analyze the LIBOR in a theoretical framework. In the complete information game framework, they prove that there exists an interval of individual bank costs for which banks quote $l\left(x_{-i}\right)$ or $u\left(x_{-i}\right)$, depending on the sign of the exposure $\nu_{i}$ to the LIBOR. For details, also concerning the data analysis in which the predicted quote clustering around the critical quantiles is investigated, see Snider and Youle (2010). Our theorems 16 and 17 also predict a higher density of quotes around the pivotal quotes.

We set $n=5$ and prove that for all parameter constellations there exists an equilibrium in which at least one player misrepresents in a maximal way. Important to note is that we say 
a player $i$ who has exposure $\nu_{i}=0$ misquotes maximally although $i$ always quotes truthfully having a singleton as the interval of possible quotes.

Theorem 18. For all $\theta$ and all $\nu$ there exists an equilibrium $x^{*}$ in which at least one player $i$ misquotes maximally, i.e. $x_{i}^{*}=\theta_{i}+\frac{\nu_{i}}{6}$.

Proof. Suppose there is no maximal misrepresentation in equilibrium. Consider without loss of generality the highest quote that is averaged in the index. If there were exactly one or five players quoting this same quote, players would have a profitable deviation. In case of five players, deviation would be to truthtelling, in case of one player deviation would be in the direction of maximal misrepresentation. Thus at the highest averaged quote between two and four players quote the same.

Suppose first the highest averaged quote is the highest quote in total. This implies nobody of the two to four players quoting this quote has negative financial exposure, all have positive financial exposure. Consider the minimum of the maximal misrepresentation of the players who quote the highest. This becomes an equilibrium if all players who quote the same highest quote deviate to quoting this minimum.

Suppose now that there is a single highest quote. The two to four players who quote the highest averaged quote then all have negative financial exposure. If the quote of two to three players is the highest which is averaged, they have a profitable deviation to a lower quote. If the four players quote the highest averaged quote, this is the lowest quote in total. Consider the maximum of the maximal misrepresentations of these players. This becomes an equilibrium if all players who quote the same lowest quote deviate to quoting this maximum.

Theorem 18 also follows from the general theorem 20 in the next subsection in which the existence of a maximal misrepresentation equilibrium in the general Median Game and Trimmed Average Game is shown.

\subsection{Comparison of the games}

The goal of this subsection is to compare the equilibrium sets of the three games with a focus on the magnitude of possible equilibrium misrepresentation. The marginal impact of an individual 
quote of a player in the Median, the Trimmed Average and the Average Game is different. This fact allows us to prove a result which gives a bound on equilibrium misrepresentations in the different games. Denote by $m \leq n$ the number of quotes averaged in the Trimmed Average Game.

Theorem 19. The maximal equilibrium misrepresentation of player $i$ is $\frac{\nu_{i}}{2}$ in the Median Game, $\frac{\nu_{i}}{2 m}$ in the Trimmed Average Game, and $\frac{\nu_{i}}{2 n}$ in the Average Game.

Proof. It is sufficient to note that the equilibrium misrepresentation in the Median, Trimmed Average and Average Game is bounded by the potential marginal influence of a single quote.

Two things are worth mentioning. First, in the Average Game the equilibrium is unique and players always quote $\theta_{i}+\frac{\nu_{i}}{2 n}$. Second, for all parameter constellations of private values $\theta$ and financial exposure $\nu$ there exists not only an equilibrium but in particular an equilibrium in which at least one player misquotes maximally. This is clear for the Average Game and follows for the Median and the Trimmed Average Game from theorem 20. The reasoning in the proof depends neither on the number of players $n$ in the game nor the number $m$ of quotes which are averaged.

Theorem 20. In the Median Game, the Trimmed Average Game and the Average Game there exists for all $\theta$ and all $\nu$ an equilibrium in which at least one player misrepresents in a maximal way.

Proof. Consider the vector of maximal misrepresentations and order it from high to low. If the maximal misquote $x_{i}$ enters the calculation of the statistic, quote $x_{i}$. If not, change the quote in the direction of $\theta_{i}$. If the intersection of the interval of possible misrepresentations with the interval of counting quotes is empty, quote $\theta_{i}$. Else misquote maximally under the constraint that the quote enters the calculation of the statistic. All players following this quote strategy defines a Nash equilibrium in the Average Game, the Trimmed Average Game and the Median Game.

The median rule increases the marginal impact in comparison with the other two statistics. Moreover, the median rule leads to a more volatile index. The average rule in contrast has the 
property of being sensitive to extreme quotes, which may turn out to be a disadvantage in practice. In this respect the trimmed average can be seen as a reasonable compromise between the other, more extreme statistics. This is in line with the arguments of Wheatley (2012a). However, statistical analysis by Eisl et al. (2013) points out that the median rule would have been less manipulable than the trimmed average between 2006 and 2012. This difference to the results of our model is probably due to two reasons. First, our informational assumption that banks know perfectly well the private values of all banks is not satisfied in reality. Second, the Nash equilibria in the Trimmed Average Game and in the Median Game are difficult to play but the more difficult ones to play are the Nash equilibria in the Median Game. An interesting next step is to make experiments in the lab to examine which equilibria are actually played in the three related games.

\section{Conclusion}

The LIBOR scandal has made headlines throughout the world in the last months. In this paper we try to contribute to the understanding of how the banks actually manipulated the LIBOR and what modification possibilities there may exist which would allow to make the mechanism more robust against manipulation. For this, we have modelled the LIBOR fixing process as a strategic game with rational banks that individually maximize their expected utility. We have analyzed several games which are closely related to the actual LIBOR setting process. Our results make the case for several changes of the current process. The LIBOR panels should be increased whenever possible to lower the marginal impact of a single contributing bank. There are however practical problems for increasing panel bank sizes as the number of candidate banks who have as global players the necessary information to quote is limited. The LIBOR quotes of the banks should be made temporarily anonymous for two reasons. First, to prevent the quotes from being an indicator of current financial strength of the banks. Second, the immediate publication may serve the banks as a collusion device. Nevertheless, the quotes should be eventually published with delay to enable identification of financial misrepresentation. The LIBOR quotes should be based on real transactions whenever possible. To enable this, a database for actual interbank lending has to be established first. We do not recommend a 
change from the trimmed mean to the median as the equilibrium misrepresentation in the Median Game can be higher than in the Trimmed Average Game due to the higher marginal influence on the fixing. A switch from a trimmed average to the average is not robust to outliers and thus problematic to introduce although the average would also induce a lower marginal influence of a single bank.

The majority of these modification ideas supported by our modelling have already been suggested in the Wheatley Review. However, these changes can only attenuate the LIBOR problem but not qualitatively solve it. The main problem remains: There are huge financial incentives to misrepresent. Concerning our model we emphasize that it is a simplification of the actual LIBOR setting process. We quantified the financial and reputational misreporting incentives and a penalty function for misrepresentation in an adhoc way. Furthermore, our banks are extremely well informed as we assumed that the knowledge of $\theta$ is common knowledge. In reality, the information distribution between the banks and the regulators is more diffuse and the maximization problem of the individual banks is not common knowledge. This makes the LIBOR problem even harder to solve. We expect the market to move away from this interest rate benchmark. In which directions the markets will evolve and what may eventually take the place of the LIBOR is hard to tell.

However, due to the immense importance of the LIBOR rate for the financial system in general and individuals all over the world in particular it remains an important task of theoretical and practical economists to come up with ideas to guarantee that the LIBOR actually represents what it is supposed to represent or to come up with a sensible alternative.

\section{References}

R. Abrantes-Metz and D. Evans. Replacing the libor with a transparent and reliable index of interbank borrowing. Available at SSRN 2142878, 2012a.

R. Abrantes-Metz and D. Evans. Will the wheatley recommendations fix libor? CPI Antitrust Chronicle November, $2012 \mathrm{~b}$.

R.M. Abrantes-Metz, S.B. Villas-Boas, and G. Judge. Tracking the libor rate. Applied Econo- 
mics Letters, 18(10):893-899, 2011.

R.M. Abrantes-Metz, M. Kraten, A.D. Metz, and G.S. Seow. Libor manipulation? Journal of Banking \& Finance, 36(1):136-150, 2012.

W.H. Buiter. Central banks and financial crises. 2008.

J. Chen. Libor's poker: Discover interbank borrowing costs amid tacit collusion. Working Paper, 2012.

C. d'Aspremont and L.A. Gérard-Varet. Incentives and incomplete information. Journal of Public economics, 11(1):25-45, 1979.

A. Eisl, R. Jankowitsch, and M. Subrahmanyam. Are interest rate fixings fixed? an analysis of libor and euribor. An Analysis of Libor and Euribor (January 15, 2013), 2013.

J.A. Garcia. 'fixing the benchmark'-wheatley considers libor overhaul. Financial Regulation International (September, 2012), 2012.

F. Gino, S. Ayal, and D. Ariely. Contagion and differentiation in unethical behavior the effect of one bad apple on the barrel. Psychological Science, 20(3):393-398, 2009.

T. Groves. Incentives in teams. Econometrica: Journal of the Econometric Society, pages 617-631, 1973.

J. Gyntelberg and P. Wooldridge. Interbank rate fixings during the recent turmoil. BIS Quarterly Review, March, 2008.

D. Kuo, D. Skeie, and J. Vickery. A comparison of libor to other measures of bank borrowing costs. Staff Reports, Federal Reserve Bank of New York (forthcoming), 2012.

C. Snider and T. Youle. Does the LIBOR Reflect Banks' Borrowing Costs? Social Science Electronic Publishing, 2010.

C. Snider and T. Youle. The fix is in: Detecting portfolio driven manipulation of the libor. Available at SSRN 2189015, 2012.

M. Wheatley. The wheatley review of libor: Final report. HM Treasury, London, 2012a.

M. Wheatley. The wheatley review of libor: Initial discussion paper. HM Treasury, 1, 2012b. 


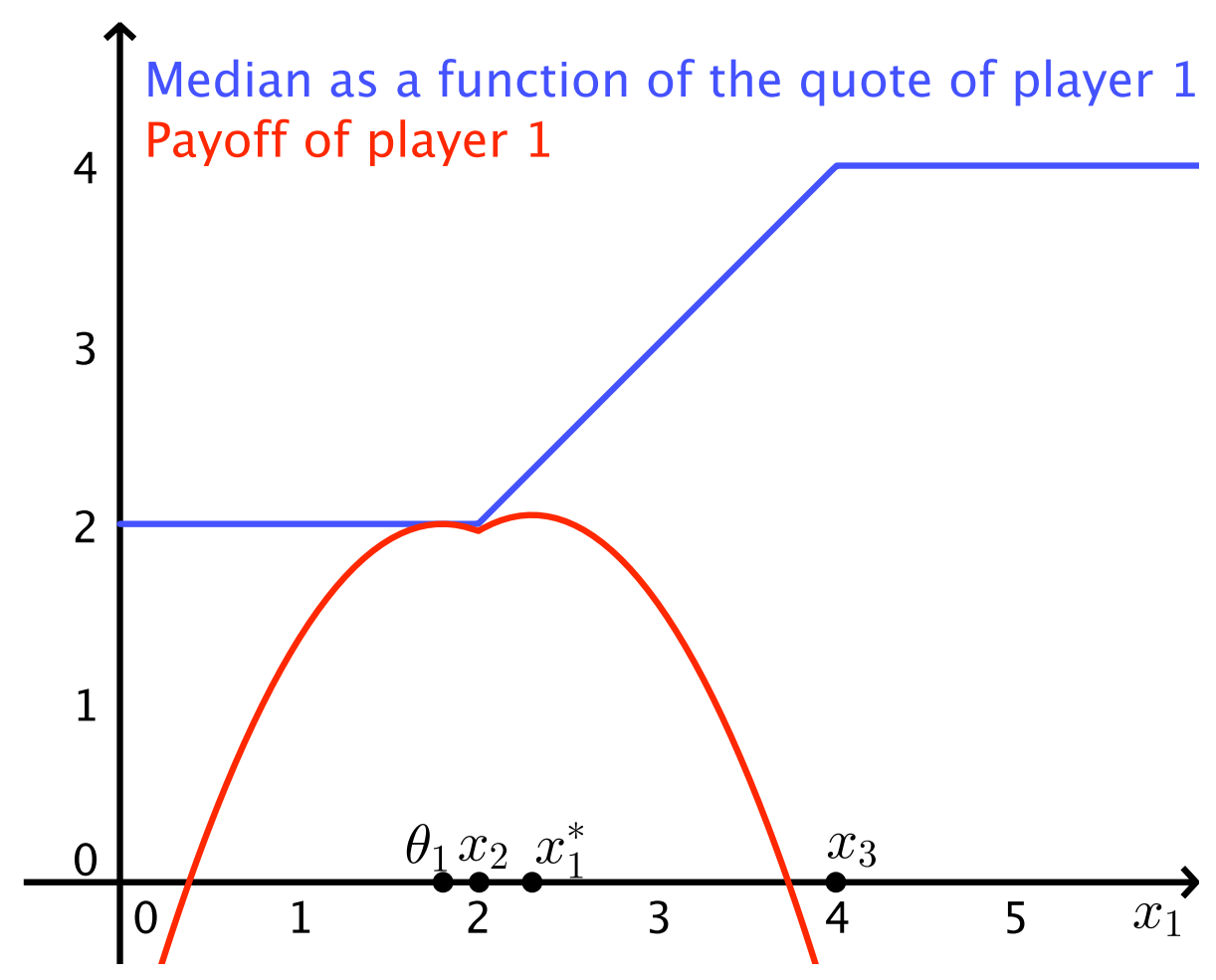

Figure 1: Example of payoff for player 1

\section{Appendix}

On figure 1. The first axis is denoted $x_{1}$ for the quote of player 1 and the second axis gives the median. $\theta_{1}$ is the private value of player 1 and $x_{2}, x_{3}$ the quotes of players 2,3 . The red line gives the payoff of player 1 as a function of player 1's quote and the blue line the median as a function of player 1's quote. $x_{1}^{*}$ maximizes the payoff of player 1 . 


\begin{tabular}{|l|l|l|}
\hline No. of Contributors & Methodology & No. of Contributor rates averaged \\
\hline 18 Contributors & top 4 highest rates, tail 4 lowest rates & 10 \\
\hline 17 Contributors & top 4 highest rates, tail 4 lowest rates & 9 \\
\hline 16 Contributors & top 4 highest rates, tail 4 lowest rates & 8 \\
\hline 15 Contributors & top 4 highest rates, tail 4 lowest rates & 7 \\
\hline 14 Contributors & top 3 highest rates, tail 3 lowest rates & 8 \\
\hline 13 Contributors & top 3 highest rates, tail 3 lowest rates & 7 \\
\hline 12 Contributors & top 3 highest rates, tail 3 lowest rates & 6 \\
\hline 11 Contributors & top 3 highest rates, tail 3 lowest rates & 5 \\
\hline 10 Contributors & top 2 highest rates, tail 2 lowest rates & 6 \\
\hline 9 Contributors & top 2 highest rates, tail 2 lowest rates & 5 \\
\hline 8 Contributors & top 2 highest rates, tail 2 lowest rates & 4 \\
\hline 7 Contributors & top highest rate, tail lowest rate & 5 \\
\hline 6 Contributors & top highest rate, tail lowest rate & 4 \\
\hline
\end{tabular}

Figure 2: Trimming rule of LIBOR

\begin{tabular}{|l|l|l|l|l|l|l|l|l|l|l|}
\hline Player & 1 & 2 & 3 & 4 & 5 & 6 & 7 & Median & Trimmed Average & Average \\
\hline Private value & 1 & 1.1 & 1.2 & 1.3 & 1.4 & 1.5 & 1.6 & 1.3 & 1.3 & 1.3 \\
\hline Quote & 1 & 1.5 & 1.2 & 1.3 & 1.4 & 1.5 & 1.6 & 1.4 & 1.38 & 1.357 \\
\hline
\end{tabular}

Figure 3: Example for influence of quotes

On figure 3: Seven players, the middle five quotes are averaged in the trimmed average. The median changes as the misrepresentation of player 2 changes the order statistic. 